coloproctology $2018 \cdot 40: 258-263$ https://doi.org/10.1007/s00053-018-0260-x Online publiziert: 4. Juni 2018

(c) Springer Medizin Verlag GmbH, ein Teil von Springer Nature 2018

CrossMark

\author{
J. Jongen ${ }^{1,2} \cdot$ V. Kahlke \\ ${ }^{1}$ Proktologische Praxis Kiel, Kiel, Deutschland \\ ${ }^{2}$ Park-Klinik Kiel, Kiel, Deutschland
}

\section{Chirurgische Therapie des Hämorrhoidalleidens}

Seit es die Menschheit gibt, wird es wahrscheinlich auch das Hämorrhoidalleiden gegeben haben. In alten Schriften werden schon Eingriffe für Hämorrhoiden beschrieben. 1882 publizierte Whitehead [21] seine Operation bei Anal- und Hämorrhoidalprolaps. Später folgten die Beschreibungen von Fansler-Anderson, Milligan-Morgan, Ferguson, Parks sowie Arnold. Im weiteren Verlauf wurden nur geringfügige Modifikationen dieser Techniken beschrieben. Erst ab den 1990er Jahren gab es die Innovation der Hämorrhoidaloperation durch Longo [10], die Klammernahthämorrhoidopexie. 1995 publizierten Morinaga et al. [13] die minimal-invasive Hämorrhoidalarterienligatur (HAL), die später um eine Mukopexie (HAL-RAR) ergänzt wurde. Im Folgenden werden diese mittlerweile bewährten Hämorrhoidaloperationen beschrieben.

\section{Prinzip der Hämorrhoidal- therapie}

Ziel der Hämorrhoidaltherapie ist nicht das Ausrotten des Hämorrhoidalplexus. Dieser ist ein physiologischer Bestandteil des Kontinenzorgans. Das Therapieprinzip ist daher die Verkleinerung des Plexus auf eine physiologische Größe sowie die Reposition und Refixation von prolabierenden Hämorrhoiden in den Analkanal. Auch für die Hämorrhoidalchirurgie gilt es, anodermschonend zu operieren; je mehr Anoderm reseziert wird, desto größer ist die Gefahr der Stenose.

\section{Exzisionsverfahren}

\section{Verfahren nach Whitehead}

Bei dem 1882 von Whitehead [21] beschriebenen Verfahren, das im deutschsprachigen Raum obsolet ist, wird das prolabierende Hämorrhoidalgewebe reseziert und die Mukosakante auf den M. sphincter ani internus in der Höhe der Anokutanlinie („anal verge“) fixiert. Ein zusätzlich bestehender Analprolaps wird einfach reseziert und nicht rekonstruiert. Durch die Fixation der Schleimhaut in Höhe der Anokutanlinie kam es zu einem Schleimhautektropion, dem WhiteheadAnus. Ein zirkuläres Vorgehen kann auch zu Stenosen in Höhe der Anokutanlinie führen, daher wird das Whitehead-Verfahren nicht mehr angewandt.

\section{Verfahren nach Milligan-Morgan}

Bei dem Verfahren, das Milligan et al. [12] 1937 veröffentlichten, wird der prolabierende Hämorrhoidalknoten nach Anlage eines perianalen Drainagedreiecks (- Abb. 1a, c) von distal nach proximal in den Analkanal vom M. sphincter ani internus abpräpariert ( $\bullet$ Abb. 1b). Oberhalb des Hämorrhoidalplexus wird eine Ligatur angelegt und das Präparat unterhalb der Ligatur abgetrennt (• Abb. 1d). Mit dieser Methode werden klassischerweise 3 Segmente exzidiert. Bei der Anlage des Drainagedreiecks können dann Marisken mitexzidiert werden. Zwischen den Resektionswunden sollten genügend breite Anoderm- und Mukosabrücken erhalten bleiben, ansonsten besteht die Gefahr der Stenose. Die Wunden wer- den nicht verschlossen, sondern der sekundären Wundheilung überlassen. Die Wundheilung nach der Hämorrhoidektomie nach Milligan-Morgan sollte idealerweise von proximal nach distal erfolgen, entsprechend wichtig ist es, dass kein Sekretstau entsteht. Dabei spielt das Drainagedreieck eine wesentliche Rolle. Es sollte groß genug sein; eine zu kleine Drainage heilt zu schnell $\mathrm{ab}$, was zu verzögerter bzw. gestörter Wundheilung führen kann. Während die Erstbeschreiber noch mit Skalpell und Schere präparierten, werden jetzt andere Techniken wie Elektrokauter, Laser, Radiofrequenz (z.B. Ligasure ${ }^{\circledR}$, Medtronic, Minneapolis, MN, USA), Ultracision ${ }^{\circledR}$ (Ethicon, Johnson\&Johnson, Norderstedt, Deutschland), usw. eingesetzt. Das Prinzip der Operation, die offene Hämorrhoidektomie, ist aber unverändert. Die Hämorrhoidektomie nach MilliganMorgan wird in Europa als Standardoperation durchgeführt.

\section{Verfahren nach Ferguson}

Bei dem Verfahren nach Ferguson [6], 1957 erstmals beschrieben, wird entsprechend der Operation nach Milligan-Morgan präpariert und reseziert. Die Wunde wird aber verschlossen. In der Originalpublikation wird die Wunde fortlaufend von kranial nach distal komplett verschlossen. Inwieweit diese geschlossene Wunde dann primär verheilt, wird kontrovers diskutiert. In vielen Fällen ist nach Ablauf von einigen Tagen eine Nahtdehiszenz zu beobachten [20]. Manche Chirurgen belassen die perianale Wunde offen und verschließen nur die in- 


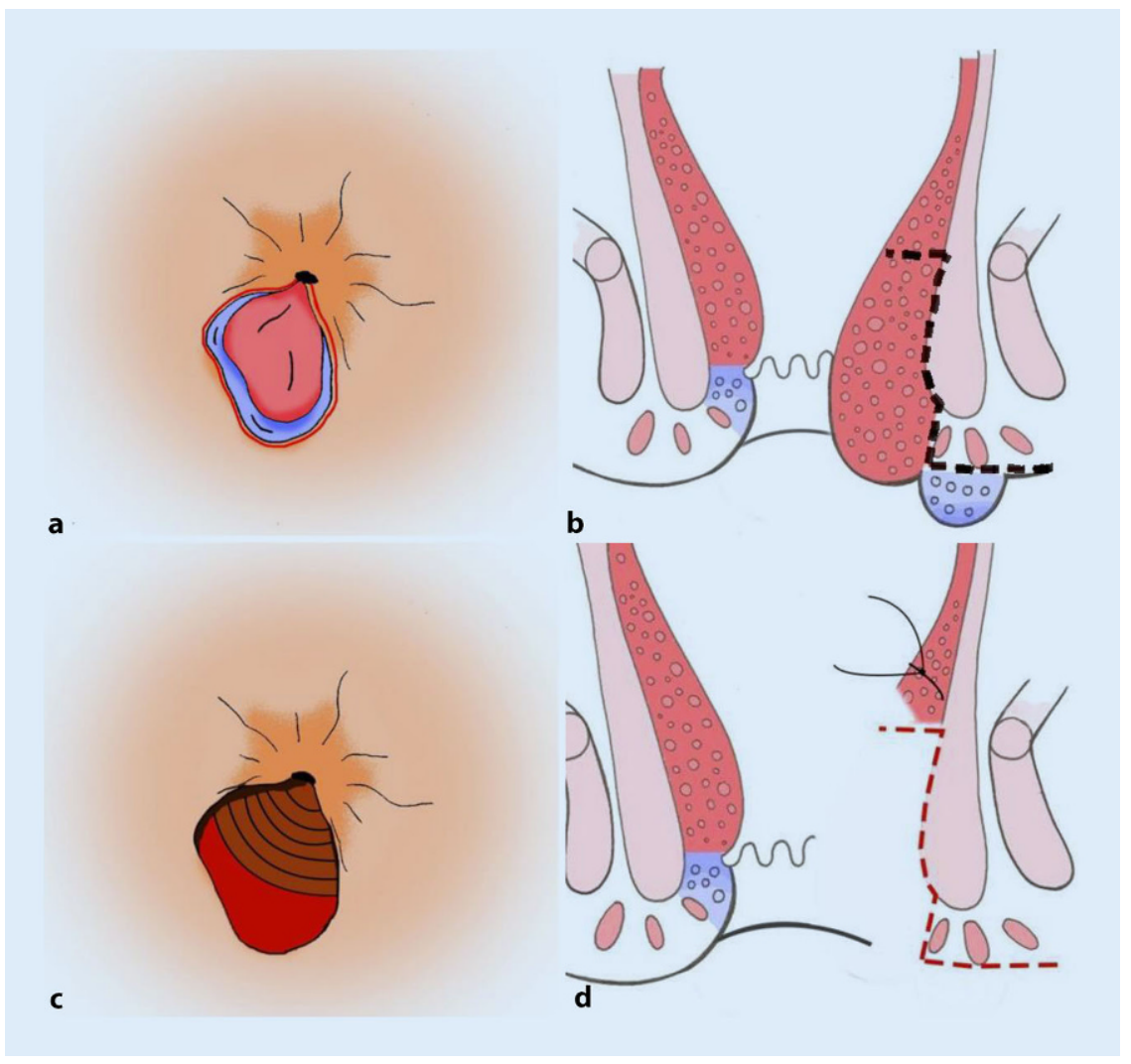

Abb. 1 ॥ Hämorrhoidektomie nach Milligan-Morgan. a Situs nach kaudal, Anlage eines perianalen Drainagedreiecks. b Längsschnitt Schnittführung/Präparation von perianal nach intraanal auf dem M. sphincter ani internus. c Wunde am Ende der Operation, Situs nach kaudal, braun: freiliegender M. sphincter ani internus, rot: Drainagedreieck. d Längsschnitt intraanale Wunde nach Ligatur und Absetzen des Hämorrhoidalknotens

traanale Wunde. Die Hämorrhoidektomie nach Ferguson gilt als geschlossene Hämorrhoidektomie und wird in den USA als Standardoperation für den Hämorrhoidalprolaps durchgeführt. Sie gilt dort gegenüber der offenen Hämorrhoidektomie als schmerzärmer. Die Hämorrhoidektomie mit einem linearen Klammernahtgerät hat sich im Alltag nicht durchgesetzt, obwohl es sich bei dieser Methode um ein geschlossenes Verfahren handelt. Auch die Durchführung in der Radiofrequenz- oder Versiegelungstechnik kann als geschlossene Hämorrhoidektomie angesehen werden.

\section{Pexierende Verfahren}

\section{Hämorrhoidalarterienligatur mit "rectoanal repair"}

Bei der Hämorrhoidalarterienligatur mit „rectoanal repair“ (HAL-RAR) handelt es sich nicht um eine Hämorrhoidek- alleinige HAL hatte auf Dauer offensichtlich nicht den gewünschten Erfolg bezüglich der Symptome, sodass die RAR oder Mukopexie zusätzlich zur HAL durchgeführt wird (nach $[2,9]$ ).

》) Die Mukopexie kann in jedem prolabierenden Teil durchgeführt werden

Nach Abschluss der HAL wird mit dem lang gelassenen Ligaturfaden die distal der HAL gelegene prolabierende Mukosa spiralförmig bis zur Linea dentata gefasst (- Abb. 2c) und dann geknotet. Dies führt zu einer Raffung und Reposition des Prolapses und demnach der für die Feinkontinenz so wichtigen Hämorrhoidalzone (• Abb. 2d). Die Mukopexie kann in jedem prolabierenden Teil durchgeführt werden. Ob eine alleinige RAR bzw. Mukopexie (ohne HAL) ausreichen kann, wird diskutiert, zumal es auch pathophysiologische Studien gibt, die nachweisen, dass der Effekt einer Hämorrhoidaloperation nicht so sehr durch eine Unterbrechung des arteriellen Zuflusses bedingt wird, sondern vielmehr durch eine Verbesserung des venösen Abflusses des Hämorrhoidalplexus [1].

\section{Klammernahthämorrhoidopexie} Morinaga et al. [13] publizierten 1995 das HAL-Verfahren. Mit einer Dopplersonde, montiert in einem Proktoskop, werden die submukös verlaufenden Hämorrhoidalarterien aufgesucht. Nach Auffinden der Arterie wird diese ligiert (- Abb. 2a). Über ein Fenster, das proximal der Dopplersonde liegt, wird das Gefäß in einer definierten Tiefe (nicht zu tief und nicht zu oberflächig) 2-fach umstochen und dann ligiert (• Abb. 2b). Auf diese Weise werden alle gefundenen Hämorrhoidalarterien ligiert. Die Anzahl ligierter Arterien kann variieren, in einigen Publikationen wird berichtet, dass die HAL bei 1, 3, 5, 7, 9 und $11 \mathrm{Uhr}$ in Steinschnittlage erfolgte. Inwieweit die Verwendung der Dopplersonde notwendig ist, wird kontrovers diskutiert. Bei der "hemorrhoid laser procedure“" (HeLP) wird die mithilfe der Dopplersonde gefundene Hämorrhoidalarterie mit einem Laserimpuls koaguliert. Die
Obwohl schon 1981 von Koblandin [9] beschrieben, hat sich erst mit Longo [10] die Klammernahthämorrhoidopexie (mit einem zirkulären Klammernahtgerät oder Stapler) großflächig verbreitet und stellt jetzt ein etabliertes Verfahren dar. Bei diesem Verfahren wird der Prolaps vollständig reponiert, bevor das Anoskop mit Obturator eingeführt wird. Nach Entfernung des Obturators wird kontrolliert, ob eine vollständige Reposition erfolgt ist. Nach Absicherung kann das Anoskop fixiert werden. Anschließend wird ein halbschalenförmiges Spekulum eingeführt und damit eine Tabaksbeutelnaht angelegt. Diese sollte etwa $3-4 \mathrm{~cm}$ proximal der Linea dentata lokalisiert sein - nicht zu hoch, damit kein „Lifting-Effekt" auftritt, und nicht zu tief, da sie sonst zu nah an der Linea dentata und am Anoderm zu liegen kommt. Sie sollte submukös 
verlaufen. Aussparungen oder Überlappungen sollten vermieden werden. Nach dem Legen der Tabaksbeutelnaht wird das geöffnete Klammernahtgerät eingeführt. Die Tabaksbeutelnaht wird auf die Achse des Geräts geknotet. Danach wird das Gerät langsam geschlossen. Bei Frauen sollte dabei die hintere Scheidewand digital und optisch mit einem Spekulum kontrolliert werden, um ein „Einklemmen“ einer Rektozele und somit eine iatrogen bedingte rektovaginale Fistel zu vermeiden. Das Gerät sollte maximal geschlossen werden, sodass die Klammern nach dem Auslösen maximal (0,75 mm) gebogen werden. Anschließend werden nach Entsicherung der Schneide- und Klammernahtvorgang ausgelöst. Dabei wird ein Ringmesser herausgefahren, das die mit der Tabaksbeutelnaht auf die Achse des Geräts fixierte Rektummukosa exzidiert. Gleichzeitig werden 2 Reihen von Klammern herausgefahren, die die Mukosa-Submukosa-Anastomose herstellen. Nach Rücksicherung wird das Gerät wieder eröffnet und aus dem Analkanal bzw. Rektum entfernt. Anschließend wird die idealerweise proximal des Hämorrhoidalplexus liegende Anastomose (wie der „doughnut“ im Gerät) auf Vollständigkeit der Naht bzw. Blutungen inspiziert. Der „doughnut“ sollte eigentlich nur Mukosa und Submukosa enthalten. Häufig finden die Pathologen aber auch Muskularisfasern, was nicht unbedingt negativ zu bewerten ist [7].

Mittlerweile stehen verschiedene Klammernahtgeräte mit eigener Gebrauchsanweisung zur Verfügung. Auch Modifikationen wurden beschrieben wie die selektive Hämorrhoidopexie mit dem zirkulären Klammernahtgerät, sog. High-Volume-Geräte usw. Pakravan et al. [14] stellten eine offene Hämorrhoidopexie ohne Klammernahtgerät vor. Dabei wird im Bereich des Prolaps eine Z-Naht gelegt, die proximale Naht kommt $4 \mathrm{~cm}$ proximal der Linea dentata, die distale Naht des Z knapp oberhalb des Hämorrhoidalplexus zu liegen. Die zwischen den Nähten gelegene Mukosa wird reseziert und der verbleibende Defekt mit der vorgelegten $\mathrm{Z}$-Naht verschlossen.

coloproctology 2018 · 40:258-263 https://doi.org/10.1007/s00053-018-0260-x

(c) Springer Medizin Verlag GmbH, ein Teil von Springer Nature 2018

\section{J. Jongen $\cdot$ V. Kahlke \\ Chirurgische Therapie des Hämorrhoidalleidens. "Same procedure as every year"?}

\section{Zusammenfassung}

Zur Therapie des Hämorrhoidalleidens stehen bewährte Methoden zur Verfügung. Diese sollen nicht als konkurrierende, sondern als sich ergänzende Verfahren angesehen werden. Beschrieben werden die Exzisionsverfahren (Hämorrhoidektomie nach Milligan-Morgan, Ferguson), die pexierenden Verfahren (Klammernahthämorrhoidopexie, Mukopexie) sowie die rekonstruktiven Verfahren nach Parks bzw. Fansler-Arnold. Darüber hinaus werden deren Stellenwert und Differenzialindikation besprochen. Dem Koloproktologen stehen verschiedene bewährte Operationen zur Therapie des Hämorrhoidalleidens zur Verfügung. Der Chirurg sollte nicht ein, sondern mehrere Verfahren anbieten können, sodass die Operation individuell auf den Befund des einzelnen Patienten zugeschnitten werden kann.

\section{Schlüsselwörter}

Hämorrhoiden · Hämorrhoidektomie $\cdot$ Hämorrhoidopexie $\cdot$ HAL-RAR . Hämorrhoidenoperation

\section{Surgical procedures for haemorrhoidal disease. Same procedure as every year?}

\section{Abstract}

Several surgical procedures for hemorrhoids have shown their efficacy. They are not competing, but complimentary procedures. The following approaches are described: excisional procedures (Milligan-Morgan and Ferguson hemorrhoidectomy), pexy procedures (stapled hemorrhoidopexy, mucopexy), and reconstructive procedures (Parks and Fansler-Arnold hemorrhoidectomy). The value and differential indication for these procedures are also discussed.
The surgeon has a whole armory to tackle haemorrhoidal disease. He should not offer only one procedure, but should have several options in order to offer a tailored approach to the findings of the individual patient. One size/procedure does not fit all.

\section{Keywords}

Hemorrhoids · Hemorrhoidectomy . Hemorrhoidopexy · HAL-RAR · Hemorrhoidal operation

\section{Rekonstruktive Verfahren}

\section{Plastische Rekonstruktion nach Fansler-Arnold}

In der Originalarbeit von Fansler u. Anderson [5] aus dem Jahr 1933 wird bei einem thrombosierten Hämorrhoidalprolaps dort, wo die prolabierte Linea dentata hätte liegen bleiben sollen, eine zirkuläre Inzision durchgeführt (• Abb. 3a). Anschließend wird der Prolaps aus dem Analkanal von proximal nach distal herauspräpariert (๑Abb. 3b). Nun wird das Anoderm vom dem sich darauf befindenden Gewebe befreit (• Abb. 3c) und überschüssige Schleimhaut reseziert. Das Anoderm wird jetzt in Form einer distal gestielten Verschiebelappenplastik nach proximal eingeschlagen (• Abb. 3d) und mit der Mukosa in Höhe der ehemaligen Linea dentata vernäht. Dabei werden Anoderm, M. sphincter ani internus und Mukosa genäht (• Abb. 3e). Somit entsteht eine Art neue Linea dentata, die sich durch die Naht des M. sphincter ani internus narbig fixiert. Letztendlich ist diese Methode eine Modifikation der Hämorrhoidektomie nach Whitehead. Die Modifikation nach Arnold [16] beinhaltet kein zirkuläres Herauspräparieren des Prolapses, sondern es werden mehrere Segmente bzw. Läppchen gebildet. Nachteil der schmalen Läppchen ist die mögliche Nekrose, daher sollten beim Verfahren nach Fansler-Arnold genügend breite Anodermbrücken stehen bleiben, sodass es im Fall einer Läppchennekrose nicht zu einer Stenose kommen kann. 


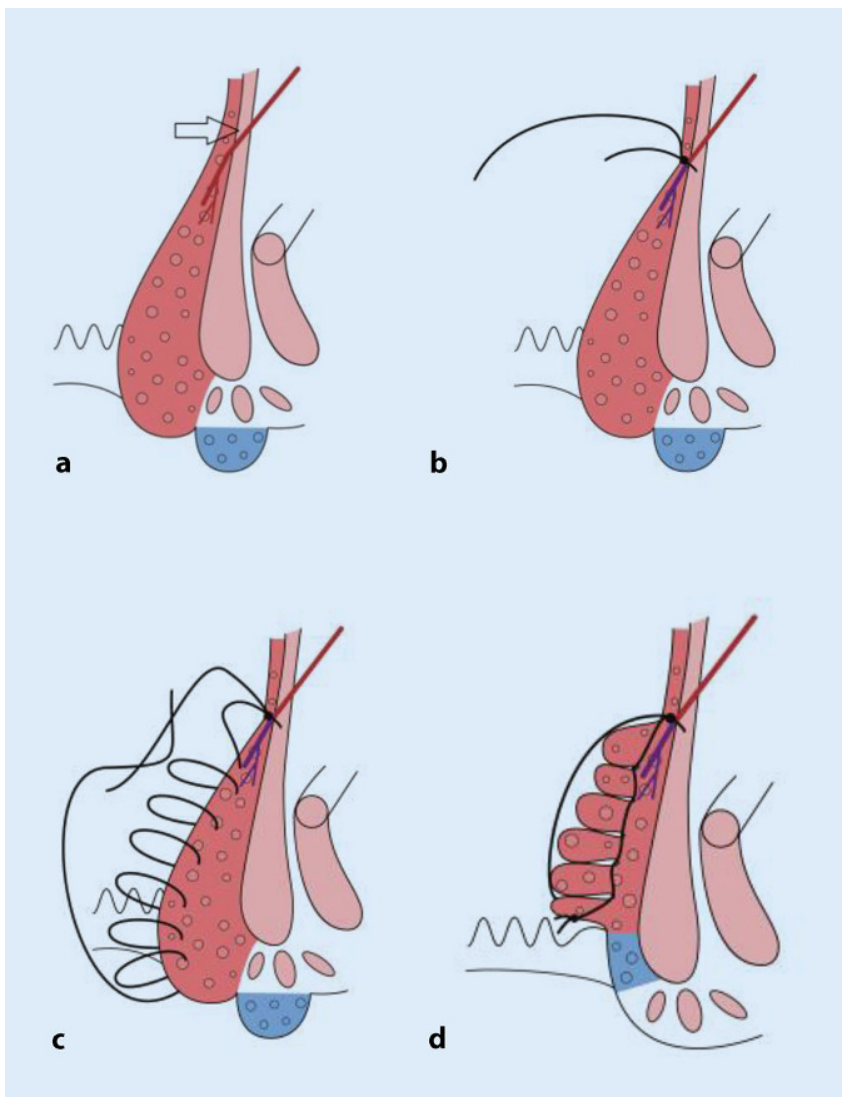

Abb. $2<$ Längsschnitt Hämorrhoidalarterienligatur mit "rectoanal repair" (HAL-RAR). a Aufsuchen der submukös verlaufenden Hämorrhoidalarterie (Pfeil). b Nach Ligatur der Hämorrhoidalarterie. c Raffung der prolabierenden $\mathrm{Mu}$ kosa distal der Ligatur. d Nach Knoten der Raffnaht

\section{Submuköse Hämorrhoidektomie nach Parks}

In der Originalarbeit von 1959 beschreibt Parks [15] seine geschlossene, submuköse Hämorrhoidektomie als ein im Vergleich zur offenen Hämorrhoidektomie schmerzärmeres Verfahren. Im Mukosabereich wird eine Längsinzision durchgeführt. In Höhe der Linea dentata wird dann nach distal das Anoderm rautenförmig inzidiert. Ausgehend von dieser Hautraute wird jetzt das prolabierte Hämorrhoidalgewebe auf dem M. sphincter ani internus nach proximal submukös präpariert. Dabei wird ausgehend von der Längsinzision die Mukosa beidseitig unterminiert, sodass dreieckförmige Mukosaläppchen entstehen. Nach ausreichender Präparation nach proximal wird das Hämorrhoidalgewebe proximal ligiert und abgesetzt. Die Längsinzision wird dann wieder verschlossen. Prolabierendes Anoderm kann dabei auch wieder in den Analkanal eingeschwenkt und in Höhe der Linea dentata fixiert werden. Mittlerweile hat es auch bei dieser Methode Modifikationen gegeben.

\section{Indikationen}

Wichtig bei der Differenzialtherapie des Hämorrhoidalleidens ist es, das Verfahren an den Individualbefund des Patienten und nicht den Befund der Operationsmethode anzupassen [22]. Es gibt keine Operation, die bei allen Befunden zufriedenstellend eingesetzt werden kann [5]. Dies bedeutet, dass der Operateur dem Patienten mehr als eine Methode anbieten können sollte. Darüber hinaus sollen die verschiedenen Operationsmethoden nicht als konkurrierende, sondern als ergänzende Verfahren angesehen werden.

\section{Zirkuläre Befunde}

Hämorrhoiden Grad 2 und 3 (reponibel). Sie sind die ideale Indikation für die Klammernahthämorrhoidopexie. Einzelne Marisken oder ein Restprolaps können abgetragen werden, ohne dass dies vermehrt Schmerzen postoperativ auslöst. Auch die HAL-RAR-Methode kann bei den zirkulären Befunden eingesetzt werden, allerdings lassen sich
Marisken mit den Raffnähten nicht beseitigen, sondern müssten dann separat exzidiert werden.

Hämorrhoiden Grad 4 (fixiert oder thrombosiert). In diesen Fällen ist ein Rekonstruktionsverfahren indiziert, entweder nach Parks oder nach FanslerArnold. Marisken werden mit diesen Verfahren ebenfalls beseitigt. Die Klammernahthämorrhoidopexie und die HALRAR-Methode sind für diese zirkulären und fixierten Befunde nicht geeignet. Generell sollte bei thrombosierten Befunden zunächst eher konservativ vorgegangen werden, da es bei den akuten, ödematösen Befunden zu einem großen Gewebeverlust und konsekutiven Stenosen kommen kann.

\section{Segmentäre Befunde mit und ohne Marisken}

Fallen nur segmentär Segmente vor, ist die Exzisionshämorrhoidektomie indiziert. Zusätzlich vorhandene Marisken können dann in die Exzision miteinbezogen werden. Die HAL-RAR-Methode wäre auch bei diesen Befunden möglich. Marisken lassen sich allerdings allein mit der Raffnaht nicht beseitigen.

\section{Stellenwert der einzelnen Methoden}

In Metaanalysen [11, 17] schneiden die operativen im Vergleich zu den nichtoperativen Verfahren (z. B. Gummibandligatur) zwar besser bezüglich möglicher Rezidive ab, verursachen aber mehr Komplikationen und Schmerzen sowie eine längere Rekonvaleszenz und Arbeitsunfähigkeit.

Die methodisch hochwertig angelegte HubBLe-Studie verglich die HALMethode mit der Gummiligaturtherapie [4]. Insgesamt 372 Patienten mit Hämorrhoiden Grad 2 (ca. 60\%) und Grad 3 (ca. $40 \%$ ) wurden 1:1 randomisiert und erhielten entweder ein Gummiband auf jede sichtbar vergrößerte Hämorrhoide oder wurden mittels HAL+/-RAR behandelt. Während die Gummibandligatur als (einmaliger) ambulanter Eingriff ohne Anästhesie erfolgte, wurde die HAL mit Anästhesie z.T. stationär (auch einma- 

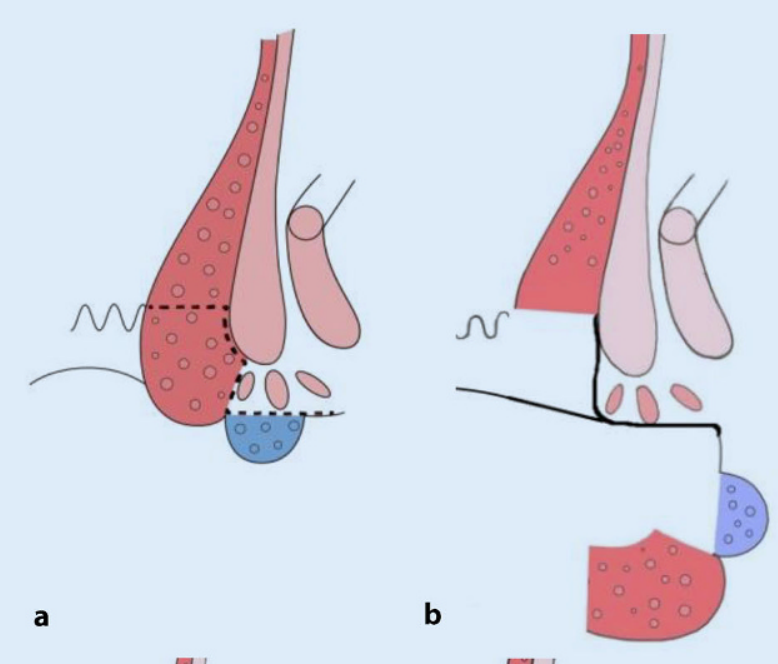

a

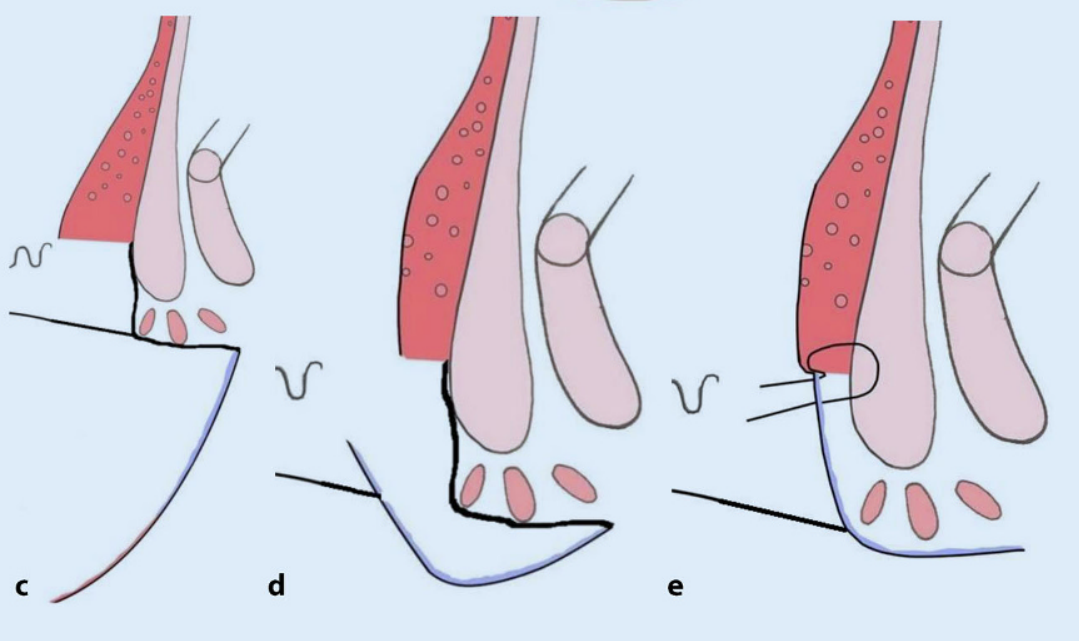

Abb. 3 ॥ Plastische Rekonstruktion des analen Kanals im Sinne einer Läppchenplastik nach Fansler-Arnold. Längsschnitt des Analkanals. a Schnittführung. b Nach dem Herauspräparieren des Prolapses von proximal nach distal. c Resektion des sich auf dem herauspräparierten Lappen befindenden Hämorrhoidalgewebes. $\mathbf{d}$ Nach Einpassen und Kürzen des präparierten Lappens. e Nach Einnähen des erhaltenen Anoderms in der Allgöwer-Donati-Technik. Kein Verschluss der Längsinzisionen

lig) durchgeführt. Primärer Endpunkt war die Rezidivrate nach 1 Jahr. Bei den Komplikationen gab es keine signifikanten Unterschiede, sie waren aber bei der HAL häufiger. Bezüglich des primären Endpunkts hatten $49 \%$ der Patienten, die mit Gummibandligatur versorgt worden waren, und $30 \%$ der Patienten mit HALBehandlung ein Rezidiv. Eine Post-hocAnalyse bereinigte die erneute Gummiligaturtherapie innerhalb des Follow-upJahres und führte zu einer Korrektur der Daten auf 37,5\% Rezidive bei diesen Patienten mit weiterhin $30 \%$ der Patienten mit HAL-Therapie. Zusammenfassend war die HAL schmerzhafter und teurer als die Gummibandligatur. Letztere zeigte die häufigeren Rezidive, allerdings nach erneutem Eingriff ähnlich gute Ergebnisse im Vergleich zur HAL+/-RAR. Nicht zu Unrecht lautete das Editorial dieser Lancet-Ausgabe: "Does HubBLe spell trouble for HAL?" [3].

In einer Metaanalyse kamen Jayaraman et al. [8] zu dem Schluss, dass die Klammernahthämorrhoidopexie gegenüber der konventionellen offenen bzw. geschlossenen Hämorrhoidektomie zu mehr Rezidiven führte. Die eTHoS-Studie [19] bestätigte dies. Allerdings muss angemerkt werden, dass sowohl in der eTHoS-Studie als auch in vielen randomisierten Studien, die in der Metaanalyse von Jayaraman et al. aufgenommen wurden, ein erheblicher Teil der operierten Hämorrhoiden dem Grad 4 zugeteilt wurde. Relativ schnell nach Einführung der Klammernahtmethode wurde aber klar, dass der fixierte Anal- und Hämorrhoidalprolaps (Hämorrhoiden Grad 4) keine Indikation für dieses Verfahren sind.

\section{》) Es sollte ein individueller Therapieansatz gewählt werden}

Simillis et al. [18] führten eine Netzwerkmetaanalyse $\mathrm{zu}$ den verschiedenen Hämorrhoidaloperationen durch. Insgesamt wurden 98 randomisierte Studien mit insgesamt 7827 Patienten untersucht und 11 verschiedene miteinander Operationen verglichen. Die Analyse ergab, dass die offene oder geschlossene Hämorrhoidektomie mehr Komplikationen und eine längere Rekonvaleszenz zur Folge haben, dafür aber weniger Rezidive. Die HAL-RAR und die Klammernahthämorrhoidopexie dagegen sind mit weniger postoperativen Schmerzen und kürzerer Rekonvaleszenz assoziiert, zeigten aber mehr Rezidive. Die Autoren empfehlen daher, die Vor- und Nachteile der angebotenen Methoden bei der Aufklärung des Patienten zu besprechen und letztendlich einen individuellen Therapieansatz zu wählen.

\section{Fazit}

Zurzeit stehen dem Chirurgen mehrere bewährte Operationsmethoden zur Verfügung. Mit diesen Methoden kann die operative Therapie auf den individuellen Hämorrhoidalbefund sowie die Wünsche und Vorstellungen des einzelnen Patienten entsprechend zugeschnitten werden. Dabei lassen sich durchaus auch mehrere Methoden miteinander kombinieren.

\section{Korrespondenzadresse}

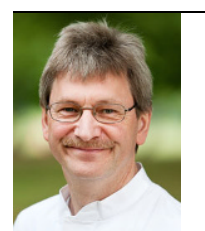

Dr. J. Jongen

Proktologische Praxis Kiel Beselerallee 67, 24105 Kiel, Deutschland info@proktologie-kiel.de

Danksagung. Die Autoren danken Andreas Scharfenstein, No Talent, Kiel, für die Erstellung der Abbildungen. 


\section{Einhaltung ethischer Richtlinien}

Interessenkonflikt. J. Jongen und V. Kahlke geben an, dass kein Interessenkonflikt besteht.

Dieser Beitrag beinhaltet keine von den Autoren durchgeführten Studien an Menschen oder Tieren.

\section{Literatur}

1. Aigner $\mathrm{F}$, Kronberger $\mathrm{I}$, Oberwalder $\mathrm{M}$ et al (2016) Doppler-guided haemorrhoidal artery ligation with suture mucopexy compared with suture mucopexy alone for the treatment of Grade III haemorrhoids: a prospective randomized controlled trial. Colorectal Dis 18:710-716

2. Awojobi OA (1983) Modified pile suture in the outpatient treatment of hemorrhoids. A preliminary report. Dis Colon Rectum 26:95-97

3. Bach SP, Fearnhead NS (2016) Does HubBLe spell trouble for HAL? Lancet 388:311-312

4. Brown SR, Tiernan JP, Watson AJM et al (2016) Haemorrhoidal artery ligation versus rubber band ligation for the management of symptomatic second-degree and third-degree haemorrhoids (HubBLe): a multicentre, open-label, randomised controlled trial. Lancet 388:356-364

5. Fansler WA, Anderson JK (1933) A plastic operation for certain types of hemorrhoids. JAMA 101:1064-1065

6. Ferguson JA, Heatin JR (1959) Closed hemorrhoidectomy. Dis Colon Rectum 2:176-179

7. Festen $S$, Molthof $H$, van Geloven AA et al (2012) Predictors of recurrence of prolapse after procedure for prolapse and haemorrhoids. Colorectal Dis 14:989-996

8. Jayaraman S, Colquhoun PH, Malthaner RA (2007) Stapled hemorrhoidopexy is associated with a higher long-term recurrence rate of internal hemorrhoids compared with conventional excisional hemorrhoid surgery. Dis Colon Rectum 50:1297-1305

9. Koblandin SN, Schalkow JL (1981) Eine neue Methode zur Behandlung von Hämorrhoiden mit Hilfe eines Zirkularstaplers. Wissenschaftliches Archiv des Zelinograder Medizinischen Institutes, Kasachstan, S27-28

10. Longo A (1998) Treatment of hemorrhoid disease by reduction in mucosal and haemorrhoidal products with a circular stapling device - new procedure. Proceedings of the $6^{\text {th }}$ World Congress of Endoscopic Surgery, Rome

11. MacRae HM, McLeod RS (1995) Comparison of hemorrhoidal treatment modalities. A metaanalysis. Dis Colon Rectum 38:687-694

12. Milligan ETC, Morgan CN, Jones LE, Officer $R$ (1937) Surgical anatomy of the anal canal, and the operative treatment of haemorrhoids. Lancet 233:1119-1124

13. Morinaga K, Hasuda K, Ikeda T (1995) A novel therapy for internal hemorrhoids: ligation of the hemorrhoidal artery with a newly devised instrument (Moricorn) in conjunction with a Doppler flowmeter. Am J Gastroenterol 90:610-613

14. Pakravan F, Helmes C, Baeten C (2009) Transanal open hemorrhoidopexy. Dis Colon Rectum 52:503-506

15. Parks AG (1959) Submucous haemorrhoidectomy. Proc RSoc Med 52(Suppl):89-91
16. Raulf F, Arnold K (1986) Stellenwert der geschlossenen Hämorrhoidektomie. Chir Gastroenterol 2:27-32

17. Shanmugam V, Thaha MA, Rabindranath KS et al (2005) Systematic review of randomized trials comparing rubber band ligation with excisional haemorrhoidectomy. Br J Surg 92:1481-1487

18. Simillis C, Thoukididou SN, Slesser AA et al (2015) Systematic review and network meta-analysis comparing clinical outcomes and effectiveness of surgical treatments for haemorrhoids. Br J Surg 102:1603-1618

19. Watson AJ, Hudson J, Wood J et al (2005) Comparison of stapled haemorrhoidopexy with traditiona excisional surgery for haemorrhoidal disease (eTHoS): a pragmatic, multicentre, randomised controlled trial. Lancet 388:2375-2385

20. Watts JM, BennettRC, Duthie HL, Goligher JC (1964) Healing and pain after haemorrhoidectomy. $\mathrm{Br} J$ Surg 51:808-817

21. Whitehead $W$ (1882) The surgical treatment of haemorrhoids. BrMed J 11:148-150

22. Windsor CJ (1965) Whitehead's operation. Aust NZ JSurg 34:311-313
Deutsches Gesundheitssystem mit Luft nach oben

Deutschland hat sein Gesundheitssystem seit der Jahrtausendwende verbessert, so eine Studie. Im Vergleich mit anderen Ländern hinkt es aber hinterher - insbesondere bei Leukämie.

Das deutsche Gesundheitssystem hat sich einer großen Studie zufolge zwischen 2000 und 2016 verbessert. Deutschland sei jedoch nicht in der Spitzengruppe aller Länder, schreiben die Forscher von der Universität Washington nach Auswertung einer globalen Gesundheitsdatenbank. Sie gaben Deutschland für 2016 mit Blick auf die Qualität und den Zugang zum Gesundheitssystem 92 von 100 Punkten. Im Jahr 2000 waren es noch 86,1 Punkte. Deutschland liegt weltweit auf Platz 18. Ganz oben auf der Rangliste stehen Island $(97,1)$, Norwegen $(96,6)$ und die Niederlande $(96,1)$. Am Ende der Tabelle finden sich vor allem afrikanische Staaten, den schlechtesten Wert erhielt die Zentralafrikanische Republik $(18,6)$. Insgesamt habe sich die Qualität und der Zugang zu den Gesundheitssystemen weltweit verbessert, heißt es in der Studie. In einigen Ländern sei die Entwicklung aber langsamer geworden, während gleichzeitig neue Anforderungen an die Gesundheitssysteme gestellt würden - etwa weil Krebs und andere nicht-übertragbare Krankheiten öfter auftreten.

\section{Leukämie-Index sehr niedrig}

Die Forscher haben für die Rangliste die Folgen von 32 Erkrankungen in 195 Ländern betrachtet, darunter unter anderem Krebs, Schlaganfälle und Epilepsie. Dabei fällt auch auf, dass in Deutschland die Bekämpfung von Leukämie noch verbesserungswürdig ist. Der Index liegt bei dieser Erkrankung bei nur 37 Punkten. Das ist weniger als in den meisten westeuropäischen Staaten bei Leukämie.

Quelle: Ärzte Zeitung basierend auf: Lanc (2018) https://doi.org/10.1016/S01406736(18)30987-5 